\title{
Multi-therapeutic effects of human adipose-derived mesenchymal stem cells on radiation-induced intestinal injury
}

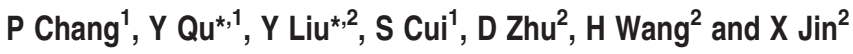

Radiation-induced intestinal injuries (RIII) commonly occur in patients who suffer from pelvic or abdominal cancer. However, current management of these injuries is ineffective. Recently, mesenchymal stem cells (MSCs) have been extensively used in regenerative medicine and have achieved a high level of efficacy. In the present study, we hypothesised that human adiposederived mesenchymal stem cells (hAd-MSCs) could be used as potential tools to heal RIll. We observed that adult SpragueDawley rats that received whole-abdominal irradiation benefitted from hAd-MSC injection. hAd-MSCs had RIIl-healing effects, including anti-inflammation, neovascularisation and maintenance of epithelium homeostasis, as indicated by elevated serum IL-10, upregulation of vascular endothelial growth factor, basic fibroblast growth factor and epidermal growth factor in irradiated intestine, mobilisation of CD31-positive haematopoietic stem cells or haematopoietic progenitor cells, and the prolonged presence of Bmi1-positive cells within crypts. Consequently, after hAd-MSC treatment, irradiated rats survived longer than non-treated animals. These results suggest that hAd-MSCs have therapeutic potential for RIII management.

Cell Death and Disease (2013) 4, e685; doi:10.1038/cddis.2013.178; published online 20 June 2013

Subject Category: Experimental Medicine

Conventional power sources and technologies have been gradually replaced by nuclear technologies due to their lesser atmospheric pollution and to the advantages of various technologies that use radiation to diagnose or treat patients suffering from abdominal or pelvic malignancies. Radiationinduced injuries are commonly observed due to the unwise use of nuclear technologies. Gastrointestinal side effects such as vomiting, diarrhoea and malnutrition frequently arise in patients with whole-body or abdomen radiation doses greater than $10 \mathrm{~Gy} .{ }^{1}$ Within the gut, intestinal stem cells (ISCs) and endothelial cells (ECs) are radiosensitive. These two types of cells are the primary targets of radiation, and the specific loss of these cells often results in a broken mucosal barrier and insufficient blood supply to the gut. ${ }^{2,3}$ Current management of radiation-induced intestinal injury (RIII) is mainly supportive and surgical, ${ }^{4}$ and there is no specific approach to treat people suffering from these injuries.

Bone marrow-derived mesenchymal stem cells (BM-MSCs) can restore epithelial renewal and absorptive functions after abdominal irradiation. ${ }^{5}$ MSCs are capable of self-replication and multi-lineage differentiation. ${ }^{6}$ Cytotherapy using MSCs may have good pro-regenerative effects on the injured epithelium, and MSCs could be ideal candidates to mediate healing of RIII. MSCs can be harvested from adipose tissue in addition to bone marrow. MSCs from these two sources present some common features, such as a spindle-like appearance, adherence to plastic when cultured and a cellular phenotype. ${ }^{7}$ Thus, we propose that adipose-derived stem cells (Ad-MSCs) might also be used to heal RIII because Ad-MSCs exhibit some superior properties compared with BM-MSCs. For example, Ad-MSCs expand faster than BM-MSCs when cultured in vitro because of higher Dkk-1 and ID-1 expression. ${ }^{8}$ In addition, the ability to suppress T-lymphocyte proliferation is higher in Ad-MSCs compared with BM-MSCs when co-cultured with peripheral blood mononuclear cells. ${ }^{9}$ Moreover, BM-MSCs must be extracted from the bone marrow, and more than a single extraction is sometimes necessary. Because subcutaneous fat is often sufficient and easy to obtain, human adipose-derived mesenchymal stem cells (hAd-MSCs) were used in the present study to evaluate their ability to heal RIII.

\section{Results}

Features of hAd-MSCs. In culture, hAd-MSCs showed a spindle-like morphology and were adherent to plastic (Figure 1A). Flow cytometry showed that hAd-MSCs were negative for CD11b, CD19, CD34 and CD45 and positive for CD73, CD90 and CD105 (Figure 1B). In addition, hAd-MSCs

\footnotetext{
${ }^{1}$ Cancer Center, The First Bethune Hospital of Jilin University, Changchun 130021, China and ${ }^{2}$ Alliancells Bioscience CO. LTD, Tianjin, 300300, China *Corresponding author: Y Qu, Cancer Center, The First Bethune Hospital of Jilin University, Changchun 130021, China. Tel: +86 13756168116; Fax: +86 431 88782488; E-mail: quyaqin52@163.com

or Y Liu, Alliancells Bioscience CO. LTD, Tianjin, China. Tel: +86 18920692888; Fax: +86 21 64085875; E-mail: andyliuliu2001@yahoo.com.cn Keywords: mesenchymal stem cells; human adipose tissue; radiation-induced intestinal injury; cell-based transplantation

Abbreviations: hAd-MSC, human adipose-derived mesenchymal stem cell; BM-MSC, bone marrow-derived mesenchymal stem cell; EC, endothelial cell; ISC, intestinal stem cell; HSC, haematopoietic stem cell; HPC, haematopoietic progenitor cell; RIII, radiation-induced intestinal injury; WAI, whole-abdominal irradiation; SDF-1, stromal cell-derived factor-1; ICAM-1, intercellular adhesion molecule-1; VCAM-1, vascular cell adhesion molecule-1; EGF, epidermal growth factor; VEGF, vascular endothelial growth factor; bFGF, basic fibroblast growth factor

Received 04.2.13; revised 23.4.13; accepted 26.4.13; Edited by RA Knight
} 

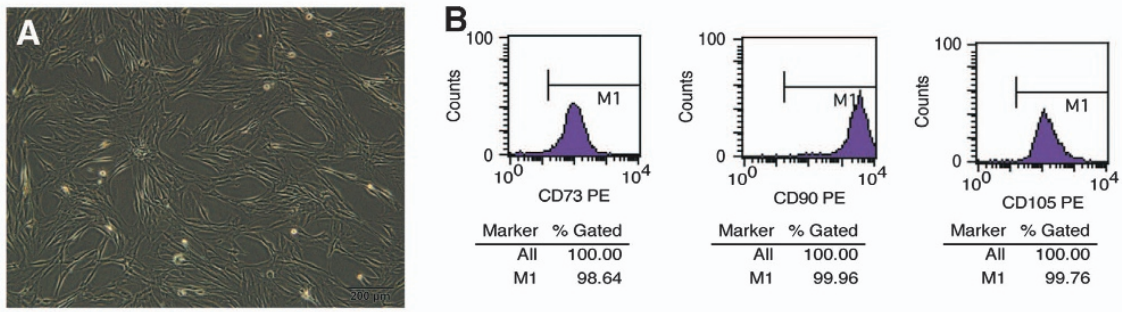

C
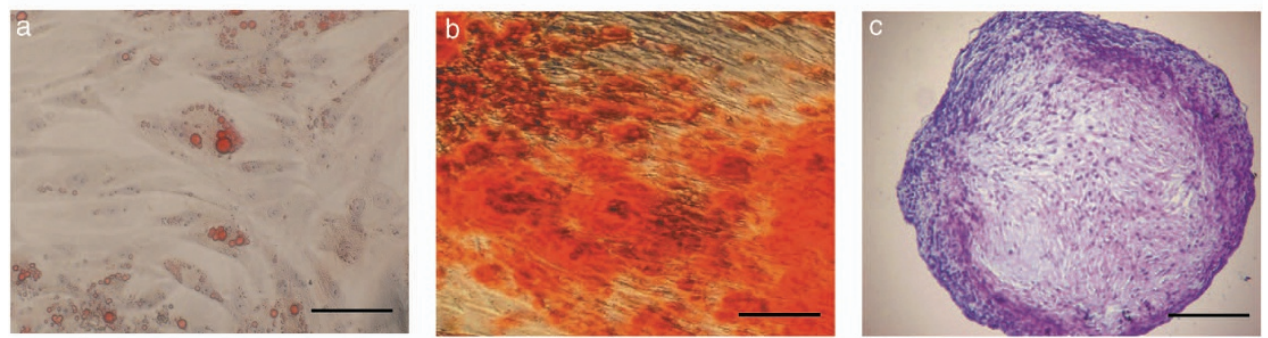

Figure 1 Features of hAd-MSCs. (A) Spindle-like shape when cultured in vitro. Scale bar, $200 \mu \mathrm{m}$. (B) Quantitative analysis by FACS. (C) Tri-lineage differentiation: (a) adipocyte, (b) osteoblast and (c) chondrocyte. Scale bar, $50 \mu \mathrm{m}$

showed tri-lineage differentiation into adipocytes, osteoblasts and chondrocytes after 21 days in defined culture media (Figure 1C). All of these results are consistent with the minimal criteria for MSC identification. ${ }^{7}$

Chemotactic property of hAd-MSCs. MSCs express specific chemokine receptors, such as CXCR4, and they migrate to radiation-injured sites by chemotaxis. ${ }^{10}$ In this study, normal rats intraperitoneally administered PBS or hAd-MSCs were used as controls to examine the chemotactic property of hAd-MSCs in the injured host. Within the first $24 \mathrm{~h}$ after abdominal irradiation, we did not observe hAdMSC migration into irradiated intestine. It was previously reported that on the 3rd day after abdominal irradiation, the percentage of MSC engraftment was significantly lower in irradiated intestines, in the range of $0.13 \pm 0.06 \% .^{5}$ In contrast, in this study, on the 10th day after hAd-MSC delivery, fluorescence imaging confirmed that numerous hAd-MSCs were implanted into irradiated intestine (Figure 2A). However, no obvious homing of hAd-MSCs was found in normal intestine (data not shown). A previous study also reported the upregulation of stromal cell-derived factor-1 (SDF-1) expression in injured sites, which was associated with elevated MSC homing efficiency due to the interaction between SDF-1 and CXCR4, a receptor expressed in MSCs. ${ }^{10}$ Furthermore, adhesion molecules, such as intercellular adhesion molecule-1 (ICAM-1) and vascular cell adhesion molecule-1 (VCAM-1), also participate in mediating the homing of MSCs. ${ }^{11}$ In this study, we observed that the expression of SDF-1 in irradiated intestines was significantly higher than that in controls on the 10th day after abdominal irradiation (Figure 2B). Moreover, after delivery of hAd-MSCs, SDF-1 expression in irradiated intestine was much higher than in the PBS-treated group (1.24-fold increase). However, there were no clear differences in the expression of ICAM-1 or VCAM-1 among groups (data not shown).

Collectively, these results demonstrate that hAd-MSCs were capable of homing to injured sites after abdominal irradiation due to the upregulation of SDF-1 by the host. To some extent, delivery of hAd-MSCs further increased SDF-1 expression within the injured sites.

hAd-MSCs improve the survival of irradiated rats and increase their body weight. Thirty rats were used in the present study: 27 rats were randomly selected to receive a lethal dose of $15 \mathrm{~Gy}$ during whole-abdomen irradiation (WAI) and 3 rats were used as controls. Of the 27 irradiated rats, 9 rats were intraperitoneally injected with hAd-MSCs (WAI + MSC group) within $2 \mathrm{~h}$ after abdominal irradiation, 9 rats were injected with human skin fibroblasts (WAI + Fibroblast group) and the remaining 9 rats were treated with PBS alone (WAI + Vehicle group). During the study, all of the rats died within 16 and 11 days after abdominal irradiation when treated with PBS and fibroblasts, respectively. In contrast, eight of nine rats survived for more than 30 days after delivery of hAd-MSCs (Figure 3a). The mean survival time of rats in the WAI + MSC group was significantly higher than in the other two groups (Figure $3 b$ ). In addition, during the first 10 days after abdominal irradiation, the ratio of body weight to initial weight showed no significant differences among the three groups. Subsequently, the ratio in the WAI + MSC group was significantly higher than that in WAI + Vehicle group. Moreover, the ratio on the 30th day reached $102.3 \%$ in the WAI + MSC group, suggesting that the mean body weight of the rats surpassed the mean body weight before abdominal irradiation; however, this ratio was still significantly lower than that in the controls (Figure 3c). 

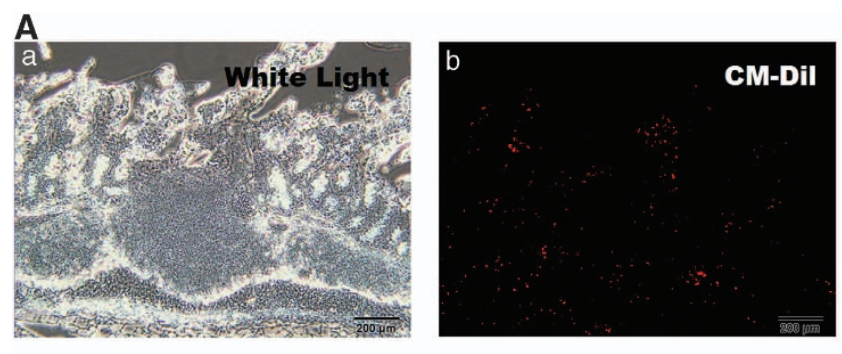

B

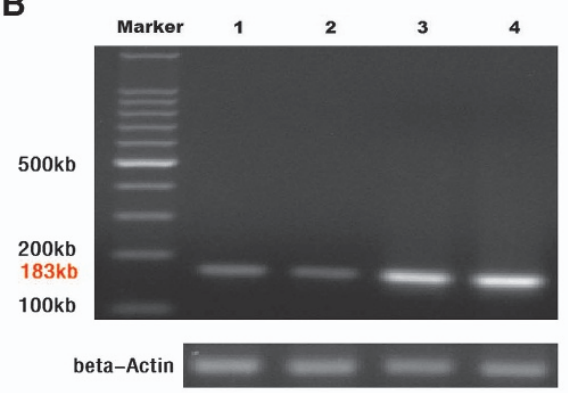

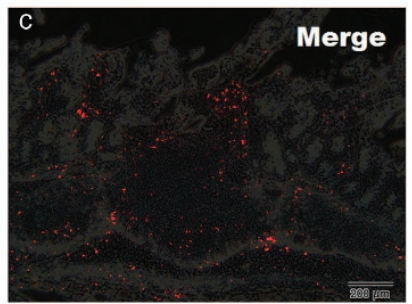

SDF-1 expression

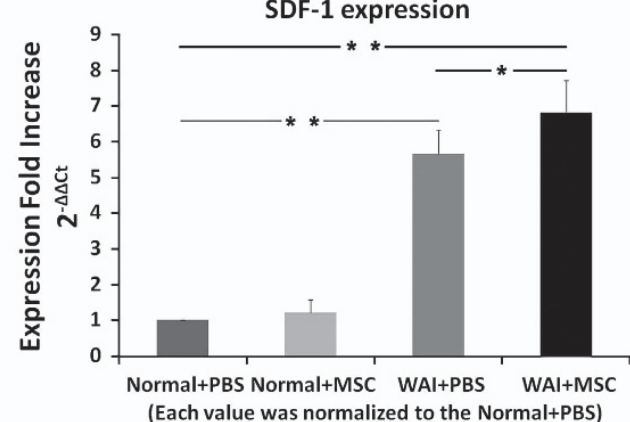

Figure 2 Chemotactic feature of hAd-MSCs. (A) Histological analysis by frozen section: (a) white light imaging, (b) fluorescent imaging and (c) merge; magnification $(\times 100)$. Scale bar, $200 \mu \mathrm{m}$. (B) mRNA expression of SDF-1 in intestine on the 10th day after abdominal irradiation. The left image represents electrophresis in 1.5\% agarose gel. Lane 1: normal + PBS, lane 2: normal + MSC and lane 3: WAI + PBS, lane 4: WAI + MSC. The right one represents quantitative analysis of SDF-1 expression. Fold expression values were normalised to normal Sprague-Dawley rat. Data represent mean \pm S.D. of three independent measurements $(n=3)$. ${ }^{\star} P \leq 0.05,{ }^{* \star} P \leq 0.001$
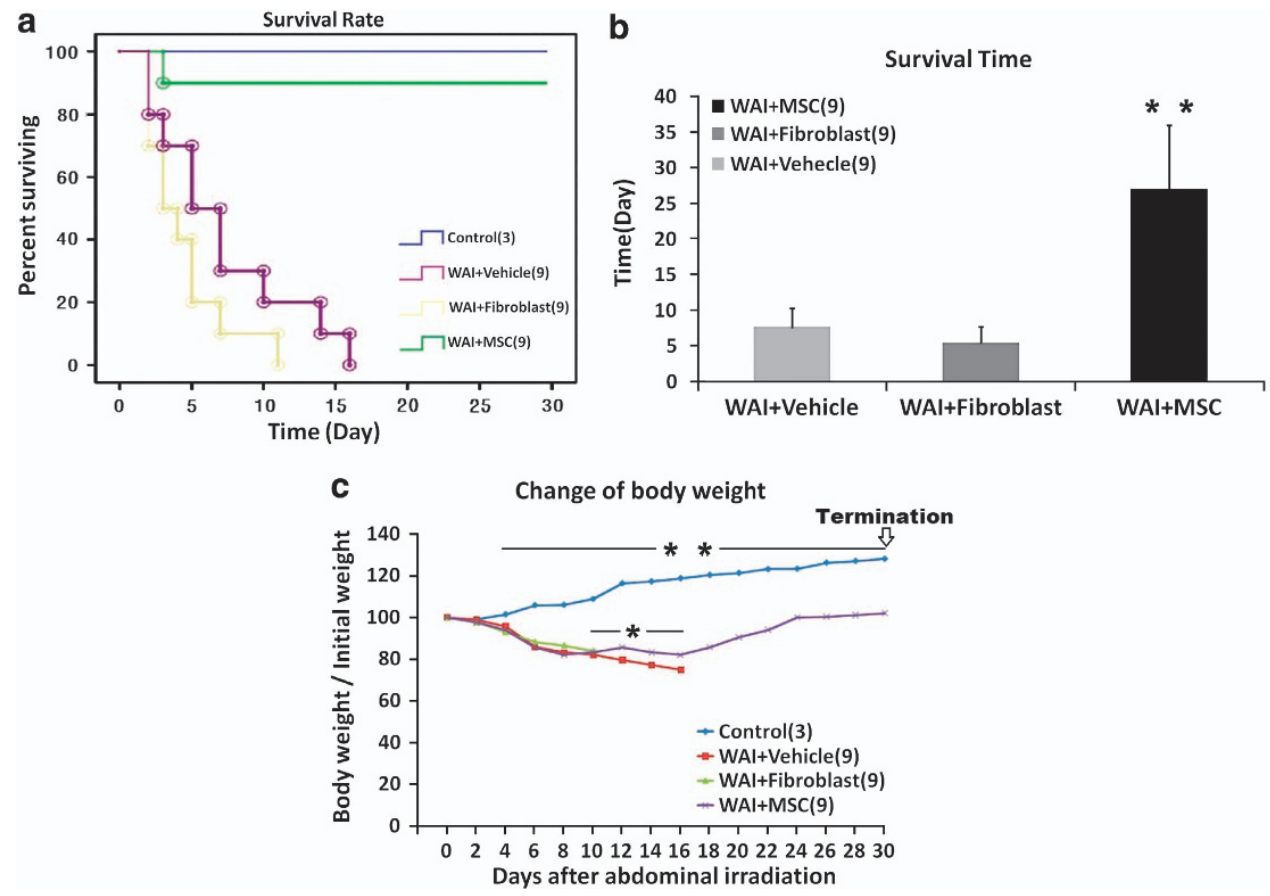

Figure 3 Delivery of hAd-MSCs increased the body weights of irradiated rats and prolonged their survival. (a) Survival rate among the four groups using Kaplan-Meier method. (b) Survival times among three groups. Data represent the mean \pm S.D. ${ }^{* \star} P \leq 0.001$ versus WAI + Vehicle group and WAI + Fibroblast group. (c) Change of body weight after abdominal irradiation. The ratio $(\%)$ of mean body weight on different days after abdominal irradiation versus the initial mean body weight before abdominal irradiation represented change of body weight on different days. ${ }^{*} P \leq 0.05$ (WAI + Vehicle group versus WAI + MSC group); ${ }^{*} P \leq 0.001$ (Control versus the rest three groups)

Inflammation decreases after delivery of hAd-MSCs. After abdominal irradiation, the RIII was evaluated every 10 days by haematoxylin and eosin (H\&E) staining. Compared with normal intestine, the epithelium was completely destroyed in the WAI + Vehicle and WAI + Fibroblast groups. Numerous leucocytes infiltrated the irradiated intestine. Moreover, in the WAI+Vehicle group, the lesion was aggravated on the 16th day after abdominal irradiation, as 
a
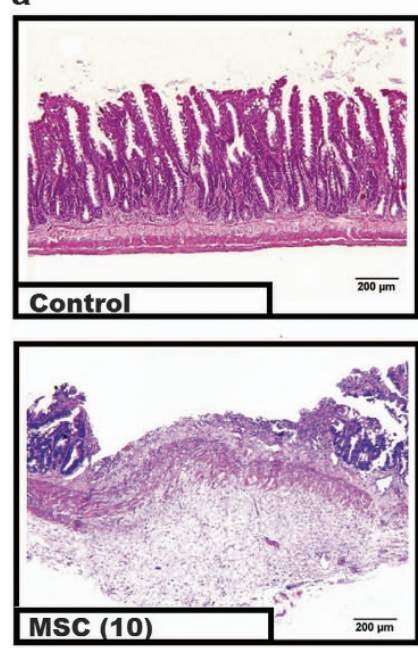

b

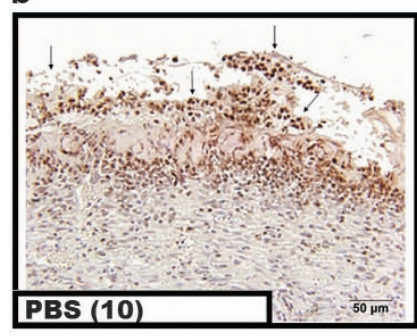

H\&E Staining
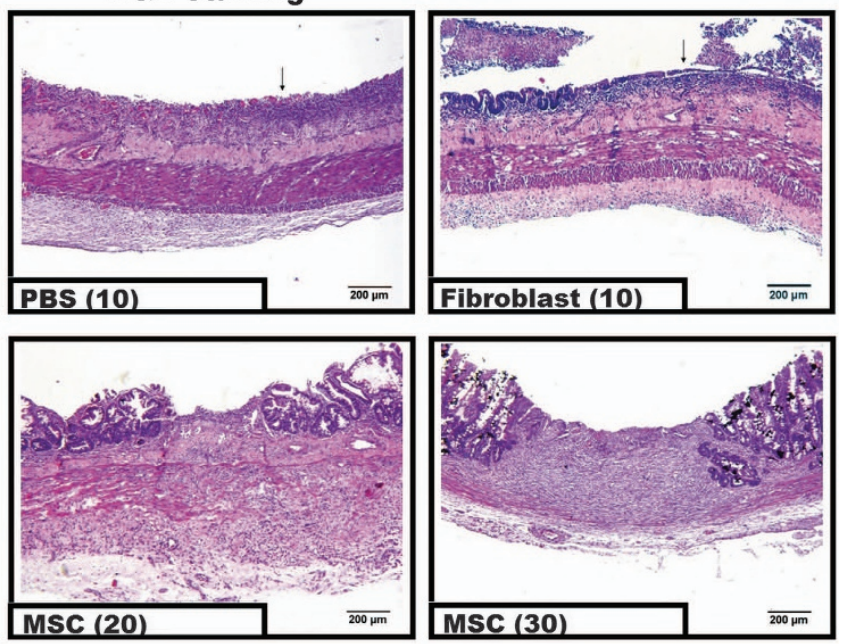
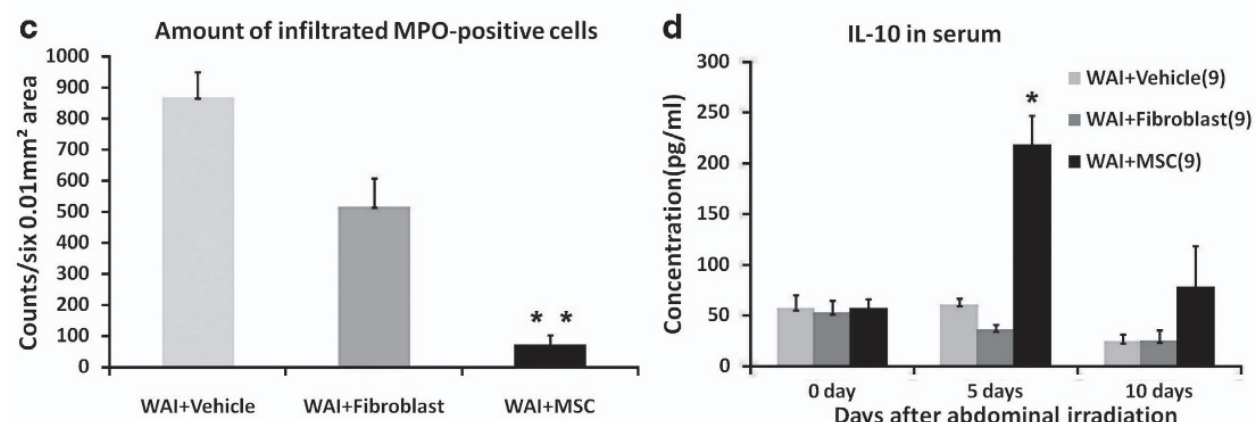

Figure 4 Delivery of hAd-MSCs decreased inflammation in the host after abdominal irradiation. (a) Histological analysis by H\&E staining. Magnification at $\times 100$. Scale bar, $200 \mu \mathrm{m}$. Black arrow represents infiltration of leucocytes. (b) Histological analysis by IHC staining with MPO antibody. Magnification at $\times 400$. Scale bar, $50 \mu \mathrm{m}$. Black arrow represents MPO-positive cell. (c) Quantitative analysis using the Image-Pro Plus 6.0 software. Data represent the mean \pm S.D. of three independent measurements $(n=3) .{ }^{* *} P \leq 0.001$ versus WAI + Vehicle group and WAI + Fibroblast group. (d) The levels of IL-10 in serum were detected by ELISA assay every five days after abdominal irradiation. Data represent the mean \pm S.D. of three independent measurements $(n=3)$. ${ }^{*} P \leq 0.05$ versus WAI + Vehicle group and WAI + Fibroblast group

detected by ecpyesis within the injured sites (data not shown). In contrast, in the WAI + MSC group, infiltration of leucocytes was remarkably mitigated within 30 days and the injured sites were surrounded by villi (Figure 4a).

Myeloperoxidase (MPO) immunohistochemistry (IHC) was used to identify the type of infiltrated leucocytes. We observed that MPO-positive cells were the most abundant cell type among infiltrating leucocytes, suggesting that infiltration by polymorphonuclear cells, such as neutrophils, increased within the injured intestine (Figure 4b). We then selected sections on the 10th day after abdominal irradiation and compared the number of infiltrated MPO-positive cells within the injured sites among groups using ImagePro Plus 6.0 software (Media Cybernetics, Rockville, MD, USA). The mean number of MPO-positive cells per six nonoverlapping $0.01 \mathrm{~mm}^{2}$ areas was used to reflect the extent of local inflammation (Figure 4c). In the WAI + Vehicle and $\mathrm{WAI}+$ Fibroblast groups, the mean numbers of infiltrated MPO-positive cells were $865.0 \pm 85.6$ and $514.0 \pm 93.1$, respectively. In contrast, in the WAI + MSC group, the mean number of infiltrated MPO-positive cells within injured intestine was $70.0 \pm 24.2$ (a significant decrease of $91.9 \%$ compared with the WAI + Vehicle group and $86.4 \%$ compared with the WAI + Fibroblast group). 
The anti-inflammatory cytokine IL-10 was measured in the serum. After the delivery of PBS or fibroblasts, the rats rarely survived for more than 10 days. Thus, we measured serum IL-10 within the first 10 days after abdominal irradiation. As shown in Figure $4 \mathrm{~d}$, before abdominal irradiation, baseline $\mathrm{IL}-10$ was $56.4 \pm 13.9 \mathrm{pg} / \mathrm{ml}, \quad 52.3 \pm 12.4 \mathrm{pg} / \mathrm{ml}$ and $56.2 \pm 9.8 \mathrm{pg} / \mathrm{ml}$ in the WAI + Vehicle, WAI + Fibroblast and WAI + MSC groups, respectively. However, compared with baseline, serum IL-10 gradually decreased within 10 days after abdominal irradiation in both the WAI + Vehicle and WAI + Fibroblast groups. By contrast, on the 5th day after abdominal irradiation, serum IL-10 had significantly increased to $217.4 \pm 29.8 \mathrm{pg} / \mathrm{ml}$ in the WAI + MSC group compared with $60.2 \pm 6.6 \mathrm{pg} / \mathrm{ml}$ in the WAI + Vehicle group $(P=0.045)$ and $35.1 \pm 6.2 \mathrm{pg} / \mathrm{ml}$ in the $\mathrm{WAI}+$ Fibroblast group $(P=0.035)$. However, 5 days later, IL-10 in the WAI+MSC group decreased to $77.6 \pm 40.6 \mathrm{pg} / \mathrm{ml}$, which was not significantly different from the WAI + Vehicle and WAI + Fibroblast groups $(23.5 \pm 8.3 \mathrm{pg} / \mathrm{ml}, P=0.19$ and $24.2 \pm 11.5 \mathrm{pg} / \mathrm{ml}, P=0.20$, respectively). In addition, the number of infiltrated neutrophils within injured sites was reduced, suggesting that temporary increases in IL-10 are sufficient to inhibit inflammation.

Delivery of hAd-MSCs promotes neovascularisation in irradiated intestine. Circulatory dysfunction commonly occurs within irradiated intestines due to massive loss of ECs. ${ }^{12}$ In the present study, double immunostaining for CD31 and VWF was used to identify ECs under confocal microscopy. CD31 and VWF double-positive cells were typically located at the lamina propria and were arranged in an organised manner (Figure 5A). However, after delivery of PBS or fibroblasts, the double-positive cells almost disappeared from the intestines with incomplete epithelium, suggesting that this area was ischaemic (Figure 5A). In contrast, in the WAI + MSC group, the number of doublepositive cells implanted to these areas progressively increased during the 30-day period after hAd-MSC delivery (Figure 5A). CD105, CD31 and isolectin-B4 were used as markers to identify naive ECs in micro-blood vessels (Figure $5 \mathrm{Ba}$ ). Indices of microvascular density and the number of CD105-positive cells per $\mathrm{mm}^{2}$ were used to compare the number of micro-blood vessels and naive ECs among groups, respectively, using Image-Pro Plus 6.0 software. On the 10th day after abdominal irradiation, both the microvascular density and number of naive ECs in the WAI + MSC group were significantly higher than in the other three groups (Figures $5 \mathrm{Bb}$ and $\mathrm{c}$ ). Moreover, these two indices in the WAI + MSC group rapidly increased during the 30-day period (data not shown). To some extent, these benefits were attributed to the upregulated expression of vascular endothelial growth factor (VEGF), basic fibroblast growth factor (bFGF) and Flk-1 within irradiated intestine after delivery (Figure $5 \mathrm{C}$ ). Our data show that the expression of VEGF, bFGF and Flk-1 in the WAI + MSC group clearly increased on the 10th day after abdominal irradiation.

Next, sections from the WAI + MSC group were selected for further analysis. First, the lineage of CD31-positive cells was confirmed. Many CD31/CD34/CD45 and CD31/CD34/CD133 triple-positive cells were found in the WAI+MSC group, indicating that haematopoietic-derived cells, such as haematopoietic stem cells (HSCs) or haematopoietic progenitor cells (HPCs), were predominant in the vascular reconstruction within injured sites compared with circulating mature ECs or endothelial progenitor cells (Figures 5Da and b). ${ }^{13}$ In contrast, when treated with PBS or fibroblasts, no clear mobilisation of CD31-positive HSCs/HPCs was found within injured intestines (data not shown). A previous study also demonstrated that CD31-positive HSCs/HPCs are capable of promoting neovascularisation in vivo and differentiating into ECs when supplemented with VEGF in vitro. ${ }^{13}$ Here, we observed that CD31-positive cells exhibited the phenotype of ECs, as assessed using vWF and Flk-1 immunostaining (Figure 5Dc and d). In addition, these cells were proliferative (Figure 5De) due to the increased expression of pro-angiogenic factors, such as VEGF, within irradiated intestine.

Collectively, these results suggest that neovascularisation was triggered and accelerated by the delivery of hAd-MSCs, as indicated by the upregulation of VEGF, bFGF and Flk-1 expression and by the large number of CD31-positive HSCs/ HPCs found implanted within the injured sites.

Proliferative cells and Bmi1-positive ISCs within crypts increase after hAd-MSC delivery. Ten days after hAdMSC delivery, the villus became more hypertrophic (Figure 6A) and grew much larger than in the other three groups (Figures 6Ba). Moreover, this hypertrophic villus was still present at 30 days (data not shown). To assess whether the number of proliferative cells increased, Ki-67 was used to detect the proliferative cells within the epithelium. In normal epithelium, proliferative cells were mainly located within the crypts (Figure 6a), as demonstrated in a previous study. ${ }^{5}$ The number of proliferative cells (number of Ki-67-positive cells per six crypts) was $38 \pm 3.2$. However, in the WAI + Vehicle and WAI + Fibroblast groups, the number of proliferative cells within the crypts were $21 \pm 1.8$ and $16 \pm 0.9$, respectively (decreases of 45.7 and $57.9 \%$ compared with control, respectively). In contrast, after hAd-MSC delivery, the mean number of proliferative cells within the crypts increased to $86 \pm 5.7$ (a 2.26-fold increase compared with control) (Figure 6Bb).

A pool of Bmi1-positive ISCs exists within crypts, which can be activated if the intestine is attacked. ${ }^{14}$ In the present study, a patch of Bmi1-positive cells was mainly located at the $4+$ position of the crypts in normal intestine. However, in the $\mathrm{WAI}+$ Vehicle and WAI + Fibroblast groups, Bmi1-positive cells were rarely found within crypts. In contrast, more Bmi1positive cells were found within the crypts, 10 days after hAdMSC delivery (Figure 6A). In addition, Bmi1-positive cells were found either at the $4+$ position of crypts or at the basement of the crypts in the WAI + MSC group. A recent study $^{15}$ demonstrated that in irradiated intestines, the expression of the stem cell marker Bmi1 was increased in Paneth cells, which subsequently acquired a stem cell phenotype and converted into a proliferative state to accelerate epithelial regeneration. Bmi1-positive cells located at the basement of the crypts were most probably derived from in situ Paneth cells.

Epidermal growth factor (EGF) is essential to maintain the self-renewal of ISCs. ${ }^{16}$ On the 10th day after abdominal 
irradiation, we compared the EGF expression in the injured intestine among the groups using real-time PCR. When treated with PBS or fibroblasts, EGF expression was significantly lower than in control cells. In contrast, a 3.2fold increase in EGF expression was observed in the irradiated intestine in the WAI + MSC group compared with control (Figure 6C). Consequently, this higher level of EGF expression accelerated the restoration of epithelial integrity in the injured intestines, with more proliferative cells within the crypts.

\section{Discussion}

The present study confirmed our hypothesis that hAd-MSCs would have therapeutic effects on RIII. Semont et al. ${ }^{5}$ administered a lethal dose of 10.5 Gy to the whole body of

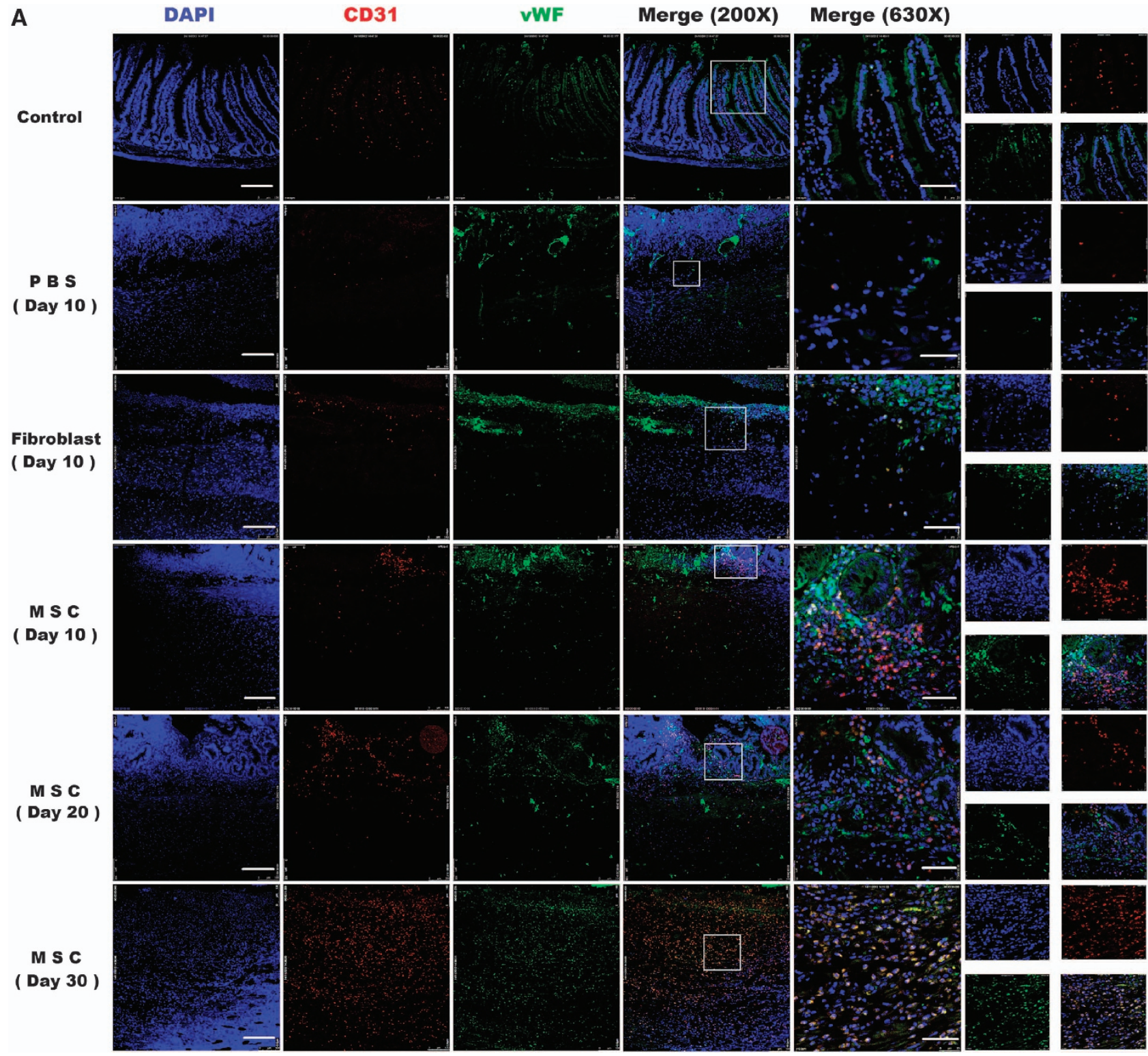

Figure 5 Delivery of hAd-MSCs promoted neovascularisation in irradiated intestine. (A) Histological analysis by IF staining. The amount of CD31 and vWF double-positive cells increased after delivery of hAd-MSCs. Magnification at $\times 630$ in the first four ranks. Scale bar, $25 \mu \mathrm{m}$. Magnification at $\times 200$ in the last two ranks. Scale bar, $100 \mu \mathrm{m}$. (B) Histological analysis by IF staining. (a) Neovascularisation in injured sites. Magnification at $\times 630$ in the first five ranks. Scale bar, $25 \mu \mathrm{m}$. Magnification at $\times 1350$ in the last two ranks. Scale bar, $10 \mu \mathrm{m}$. (b and c) Quantitative analysis of microvascular density (MVD) and the amount of naive ECs per $1 \mathrm{~mm}^{2}$ using Image-Pro Plus 6.0 software. Data represent mean \pm S.D. of six independent measurements $(n=6) .{ }^{* \star} P \leq 0.001$ versus other three groups. (C) The mRNA levels of VEGF, bFGF and Flk-1 were detected by real-time PCR 10 days after abdomial irradiation with beta-actin as the internal control. The left rank represents images of electrophresis in $1.5 \%$ agarose gel. Lane 1 : control, lane 2: WAI + PBS, lane 3: WAI + Fibroblast, lane 4: WAI + MSC. The right rank represents quantitative analysis of gene expression. Fold expression values were normalised to the control. Data represent mean \pm S.D. of three independent measurements $(n=3)$. ${ }^{*} P \leq 0.05,{ }^{* *} P \leq 0.001$. (D) Histological analysis by IF staining. (a) CD31/ CD34/CD45 triple-positive cells, (b) CD31/CD34/CD133 triple-positive cells, (c) CD31/CD34/vWF triple-positive cells, (d) CD31/CD105/Flk-1triple-positive cells and (e) CD31/ CD34/Ki-67 triple-positive cells. Magnifications at $\times 630$ and $\times 1350$. Scale bars, 25 and $10 \mu \mathrm{m}$, respectively 
B

a

Control
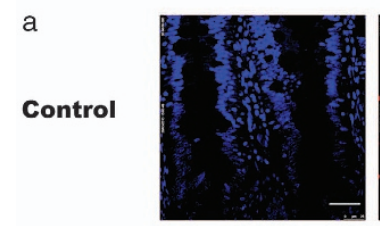

P B S

(Day 10)
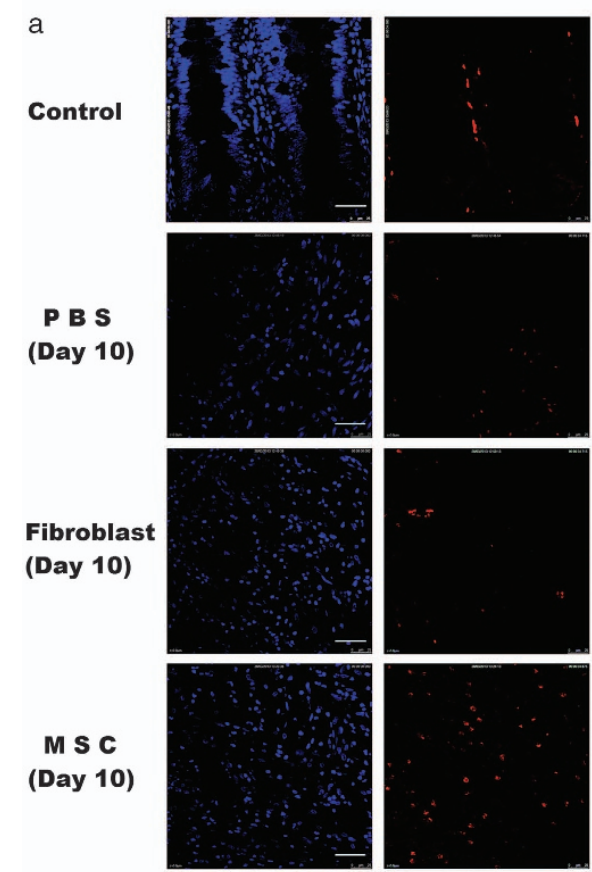

M S C

(Day 10)
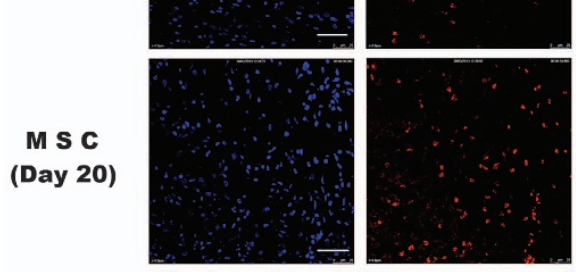

M S C

(Day 30)
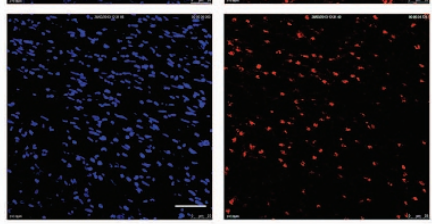

Naive ECs

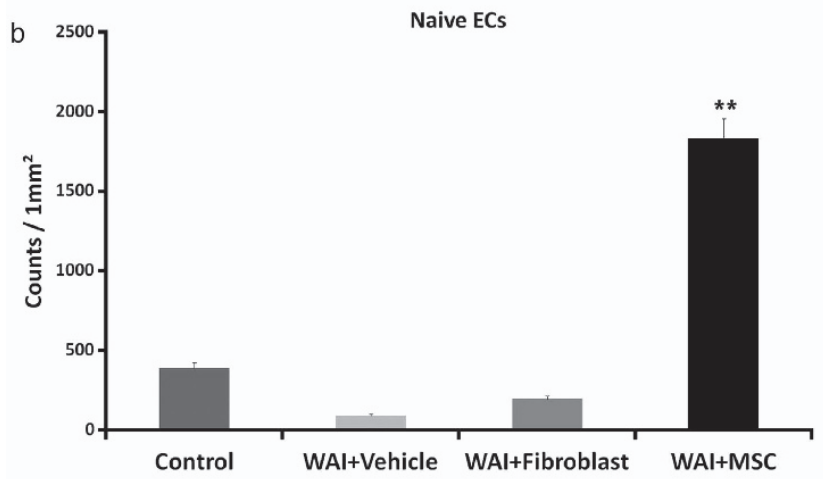

Figure 5 Continued

mice to evaluate the effect of BM-MSCs on the maintenance of homeostasis by the intestinal epithelium. Paris et al. ${ }^{17}$ found that most mice died from bone marrow failure rather than gastrointestinal syndrome if the whole body received less than $14 \mathrm{~Gy}$, and the death incidence at $14 \mathrm{~Gy}$ triggered by bone marrow failure increased up to $75 \%$. In contrast, irradiation of $15 \mathrm{~Gy}$ to the whole body in mice led to the

\section{CD105 Merge (630x) Merge (1350x)}
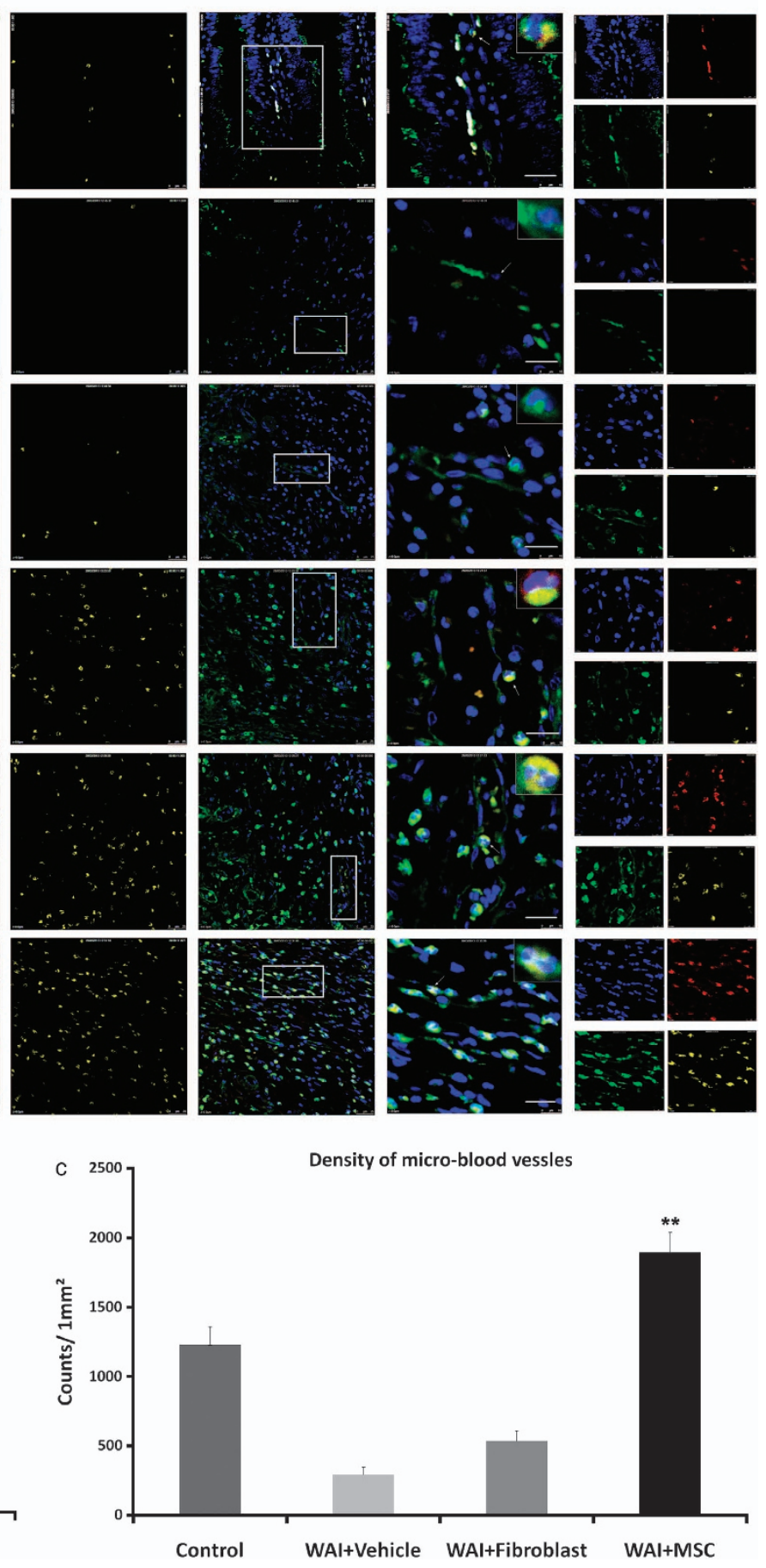

extensive loss of the villus-crypt system and initiated gastrointestinal syndrome. ${ }^{17}$ Because the loss of epithelium is dose dependent, ${ }^{1}$ our preliminary results indicated that the epithelial integrity showed large differences between 15 Gy and lesser doses, which was consistent with previous findings obtained by Paris et al. ${ }^{17}$ In addition, there was a negative linear correlation between the irradiation dose and survival 

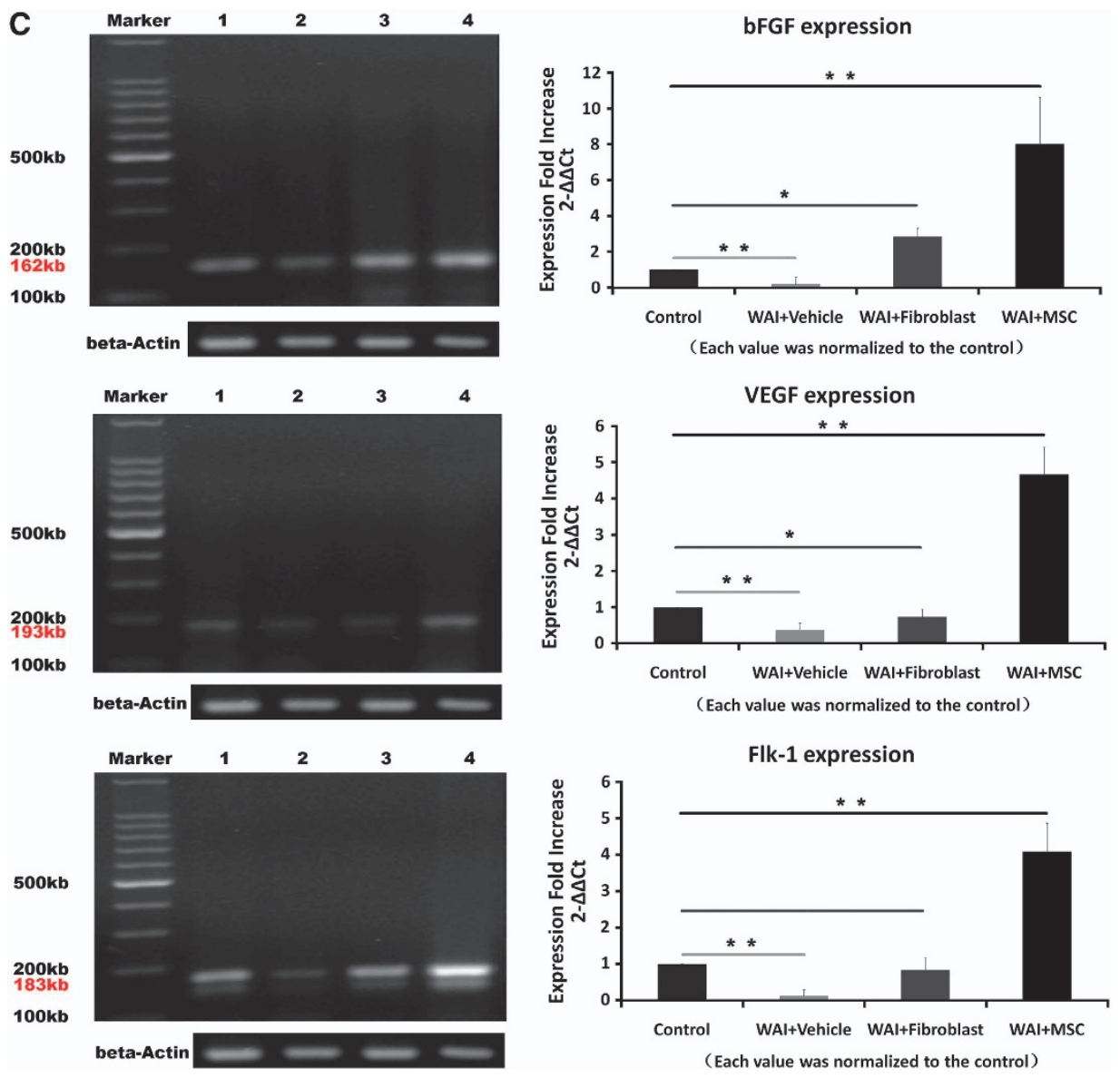

Figure 5 Continued

time of rats (Supplementary Figure 1). Thus, a lethal dose of $15 \mathrm{~Gy}$ was administered to the rats in this study. Leucocytes are sensitive to ionising irradiation. Recruitment of these cells to injured intestines was preserved by WAI, and therefore, bone marrow failure was avoidable. After abdominal irradiation, hAd-MSCs were injected within $2 \mathrm{~h}$ because leucocyte rolling peaks at $2 \mathrm{~h}$ after high-dose irradiation ${ }^{18}$ and early administration of MSCs can enhance the survival rate of irradiated animals. ${ }^{5,19,20}$ Our results reveal that therapeutic effects on RIII were not achieved by the delivery of PBS or fibroblasts and that these rats died within a short period after abdominal irradiation. In contrast, most rats were rescued by hAd-MSCs and survived to the end of the present study. The therapeutic effects of hAd-MSCs on RIII can be categorised into the following three aspects: (1) anti-inflammation, (2) promotion of neovascularisation and (3) acceleration of the restoration of epithelial integrity.

Under physiological conditions, epithelial homeostasis is maintained by proliferative cells within crypts, such as ISCs and transit-amplifying cells. ${ }^{2}$ However, injuries to proliferative cells commonly occur in irradiated intestine. Consequently, epithelial integrity is difficult to preserve due to the tremendous loss of proliferative cells over a short period of time. An incomplete epithelium also cannot easily maintain the absorptive and defensive functions of the gut. ${ }^{3}$ Thus, a rapid decline in body weight was observed among the rats in the $\mathrm{WAI}+$ Vehicle and WAI + Fibroblast groups.

As previously reported, damage to ECs initiated subsequent lesions within irradiated intestines, such as inflammation. $^{3,17}$ Moreover, local inflammation increased after exposure to enteric bacteria. ${ }^{21}$ Consequently, in the WAI + Vehicle and WAI + Fibroblast groups, persistent infiltration of neutrophils was observed within injured intestines. Neutrophils release MPO, which results in further damage to ECs. ${ }^{22}$ Massive loss of ECs leads to circulatory dysfunction within irradiated intestine, which is harmful to the nutrient-exchange function of the gut. ${ }^{23}$

In contrast, in the WAI + MSC group, inflammation was mitigated within the injured sites, and IL-10 significantly increased after hAd-MSC delivery. Soluble factors such as IL-10 can decrease inflammation in the host. On the basis of our results, high IL-10 in serum after hAd-MSC delivery might involve several mechanisms. MSCs can inhibit inflammation via autocrine IL-10 signalling. ${ }^{24}$ In addition, human MSCs release signals to several types of allogenic pro-inflammatory cells to alter their cytokine-secretion profiles. ${ }^{25}$ For example, Nemeth et al. ${ }^{26}$ reported that in septic mice, IL-10 released by host macrophages was increased after bone marrow MSC delivery because the inflammatory milieu induced MSCs to upregulate the expression of COX-2 and production of PGE2, 
D a 630X Magnification

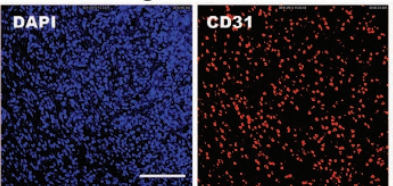

1350X Magnification

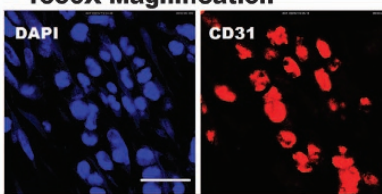

630X Magnification

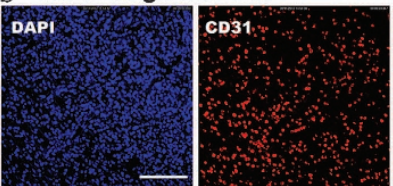

1350X Magnification
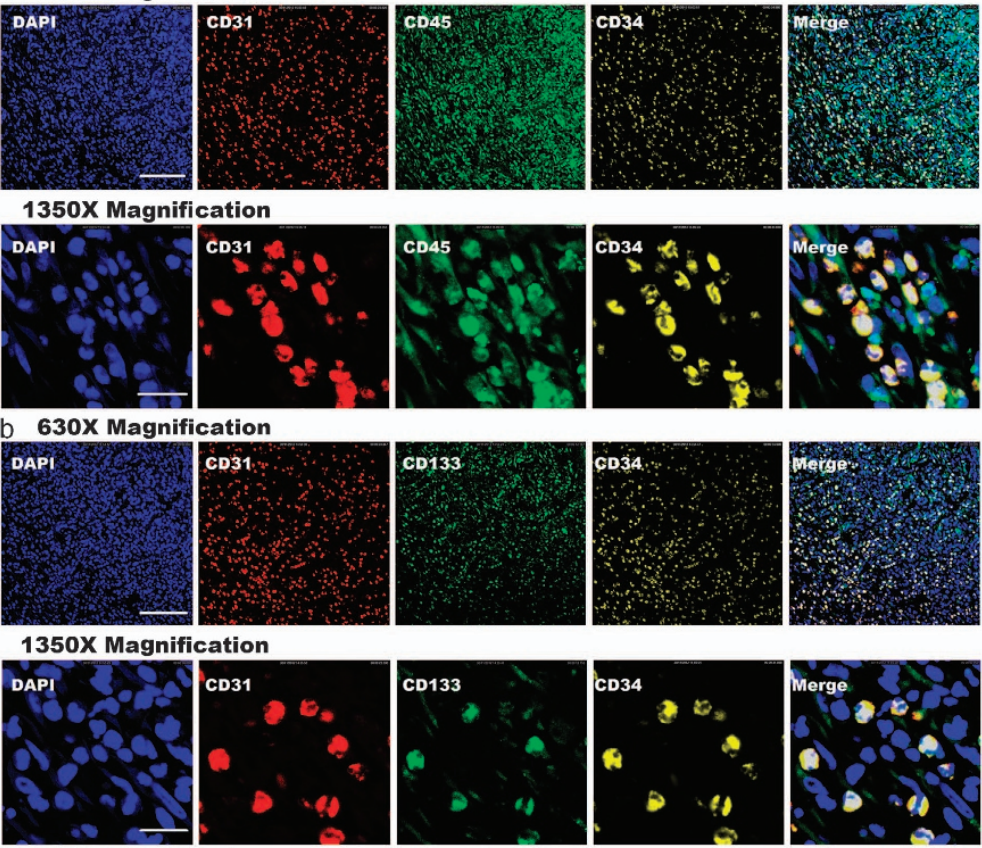

C 630X Magnification
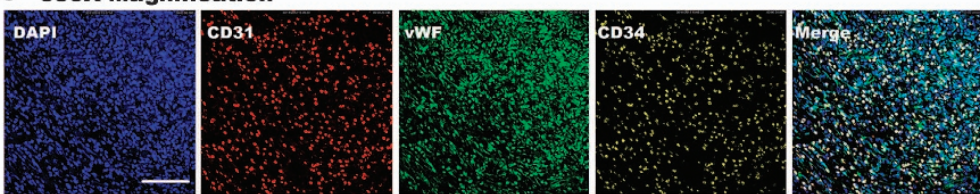

1350X Magnification
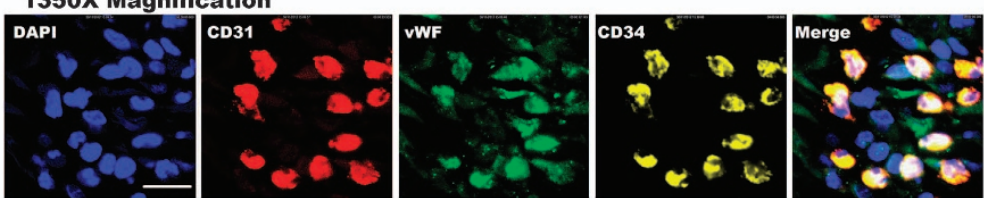

\section{d 630X Magnification}
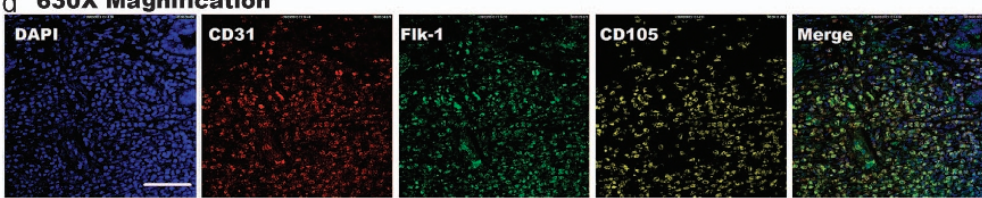

1350X Magnification
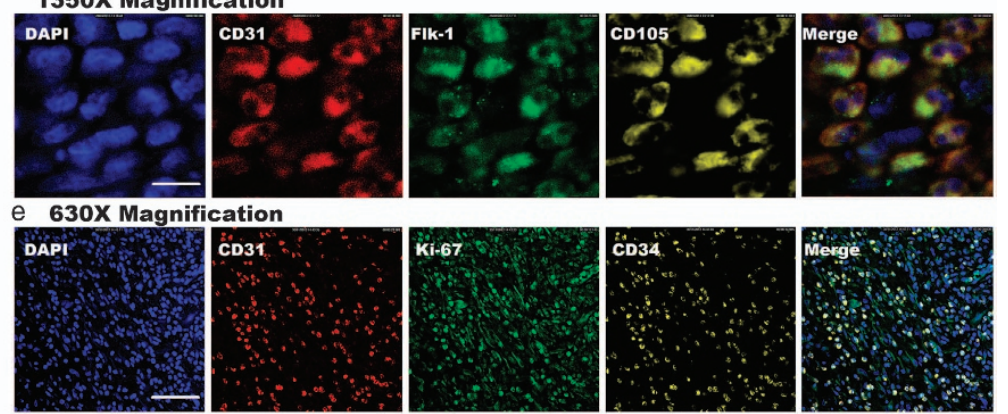

1350X Magnification
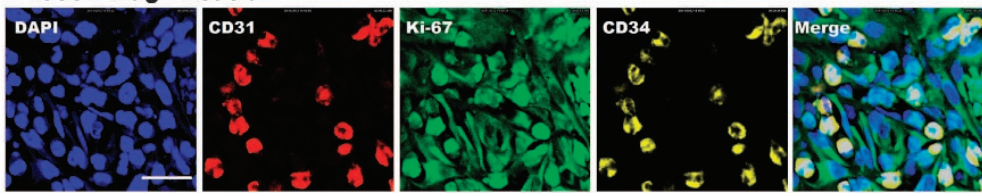

Figure 5 Continued 

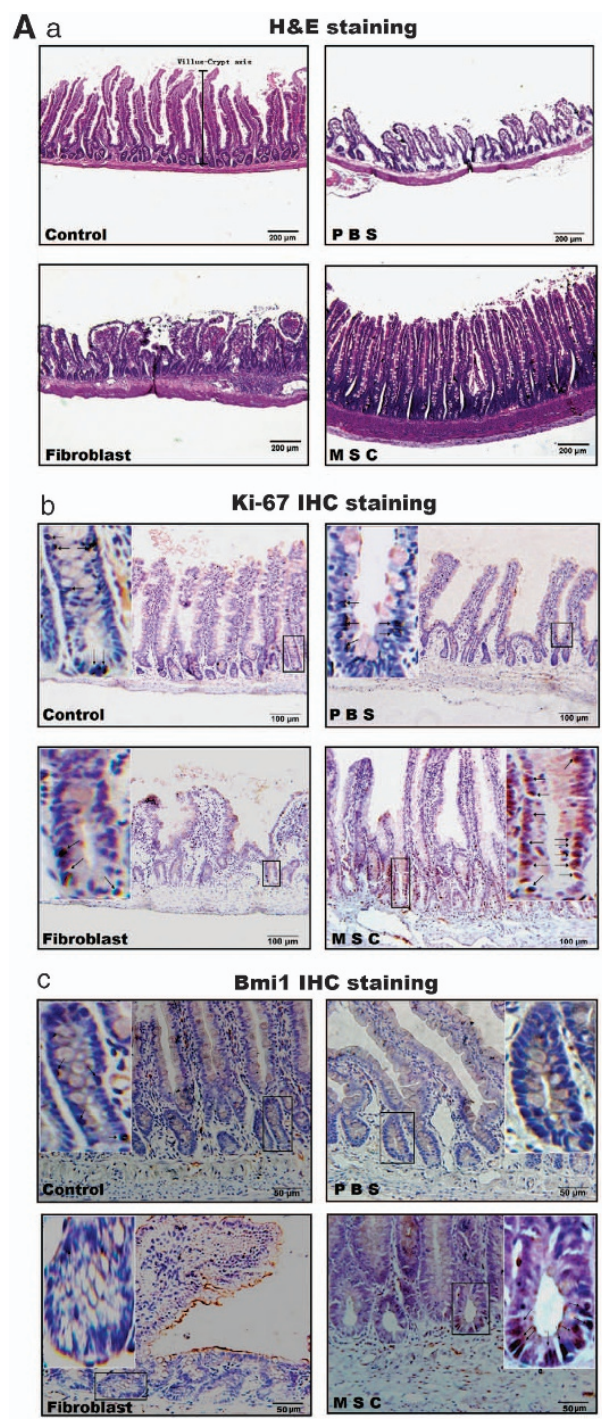

\section{B}
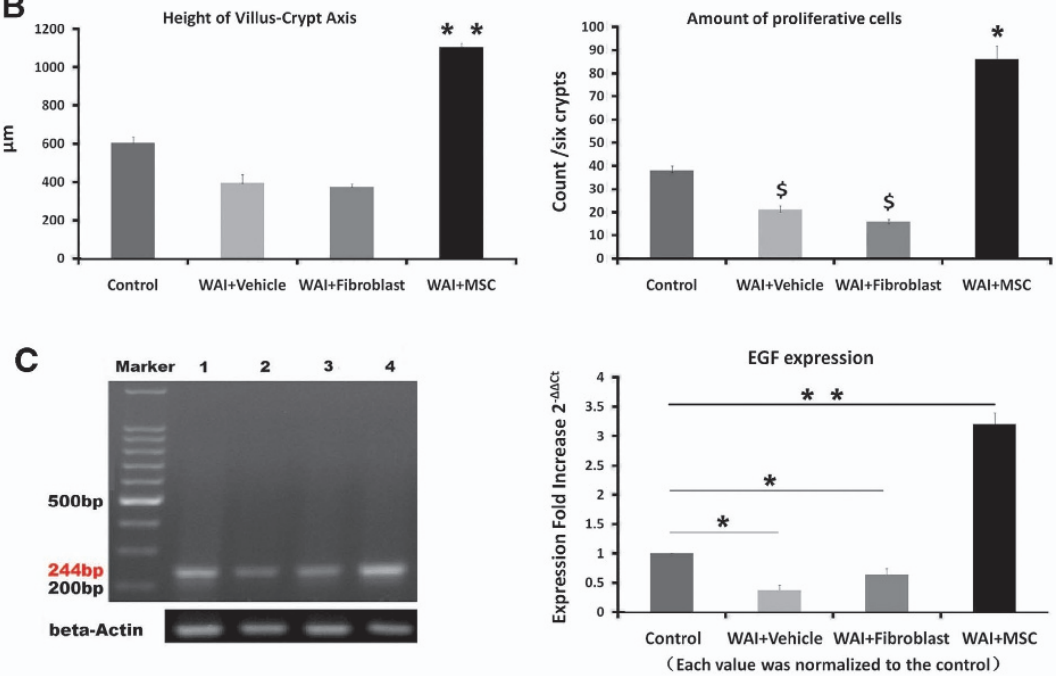

Figure 6 Delivery of hAd-MSCs promoted proliferation of epithelial cells within crypts. (A) Histological analysis. Upper panel: H\&E staining. Magnification at $\times 100$. Scale bar, $200 \mu \mathrm{m}$. Middle panel: IHC staining with Ki-67 antibody (black arrow). Magnification at $\times 200$. Scale bar, $100 \mu \mathrm{m}$. Lower panel: IHC staining with Bmi1 antibody (black arrow). Magnification at $\times 400$. Scale bar, $50 \mu \mathrm{m}$. (B) Quantitative analysis. (a) Height of villus-crypt axis among groups, (b) amount of proliferative cells in crypts. Data represent mean \pm S.D. of six independent measurements $(n=6)$. ${ }^{*} P \leq 0.05$ versus control; ${ }^{\$} P \leq 0.05$ versus control; ${ }^{\star \star} P \leq 0.001$ versus other three groups. (c) The mRNA levels of EGF were detected by real-time PCR 10 days after abdomial irradiation with beta-actin as the internal control. The left image represents electrophresis in $1.5 \%$ agarose gel. Lane 1: control, lane 2: WAI + PBS, lane 3: WAI + Fibroblast, lane 4: WAI + MSC. The right one represents quantitative analysis of EGF expression. Fold expression values were normalised to the control. Data represent mean \pm S.D. of three independent measurements $(n=3)$. ${ }^{*} P \leq 0.05,{ }^{* *} P \leq 0.001$

which subsequently bound to EP2 and EP4 receptors on macrophages to increase IL-10 release by macrophages. Gonzalez-Rey et al. ${ }^{27}$ obtained similar results and proposed the novel mechanism that hAd-MSCs induce the release of IL-10 by regulatory T cells in colitic mice. ${ }^{27}$ High IL-10 inhibits the inflammatory process, such as rolling, adhesion and transepithelial migration of neutrophils in the inflammatory host. $^{26}$ In our study, infiltration of neutrophils into injured sites was mitigated 10 days after hAd-MSC delivery, which might partially be attributed to temporary increases in serum IL-10. Moreover, this reduced number of neutrophils was sustained until the termination of the study. Consequently, a benign growth environment in the absence of oxidative stress was created for various cell types in the injured intestines, especially for ECs and epithelial cells. . $^{3,43}$

Damaged ECs were the primary cause of subsequent lesions. Recovery of ECs was important in protecting injured intestines from further damage. Thus, the number of ECs significantly increased within irradiated intestine after delivery of hAd-MSCs. Moreover, the ECs were naive, and a high density of micro-blood vessels was formed, as assessed by CD105 and isolectin-B4 staining. In addition, bone marrowderived cells, such as endothelial progenitor cells, contribute to the generation of ECs within injured intestinal tissue. ${ }^{28}$ Nevertheless, these naive ECs express CD45, a panhaematopoietic cell marker, indicating that these cells are 
not endothelial lineage derived. Moreover, we discovered that most of the haematopoietic-derived CD31-positive cells in the injured sites were positive for CD34, CD45 and CD133, suggesting that these cells were HSC- or HPC-derived. Kim et al. $^{13}$ reported that $\sim 98$ and $90 \%$ of murine HSCs and murine HPCs, respectively, are CD31-positive cells and are capable of differentiating into ECs due to their endothelialspecific protein expression, such as Flk-1, vWF and VE-cadherin, when supplemented with VEGF in vitro. Their results also suggest that HSCs and HPCs are exclusively restricted to CD31-positive bone marrow cells and that these CD31-positive cells promote neovascularisation in the ischaemic hindlimb of nude mice. ${ }^{13}$ ECs upregulate Flk-1 expression in the process of forming new blood vessels. In this study, we showed that the expression of Flk-1 within injured intestine in the WAI + MSC group was significantly increased on the 10th day after abdominal irradiation. Moreover, the expression of VEGF and bFGF by the host was also upregulated at the same time. Within injured sites, implanted MSCs upregulate their expression of MIP-1 and MIP-2, as well as MCP-5, to accelerate the recruitment of macrophages into injured sites. ${ }^{29}$ After the engulfment of apoptotic neutrophils, macrophages produce pro-angiogenic factors, such as VEGF, HGF and angiopoietin-1. ${ }^{30}$ We believe that increased levels of VEGF induce CD31-positive HSCs/HPCs to differentiate into more proliferative ECs, and that Flk-1 expression is upregulated in these proliferative cells, which, consequently, facilitate neovascularisation to injured sites. However, the present results show no clear mobilisation of CD31-positive HSCs/ HPCs to ischaemic areas after delivery of PBS or fibroblasts. Further studies are required to investigate these issues.

Under physiological conditions, Lgr5-positive ISCs are mainly located at the basement of crypts and produce proliferative cells to maintain epithelial homeostasis. ${ }^{31}$ A recent study ${ }^{14}$ showed the existence of another pool of ISCs, Bmi1-positive ISCs, within the crypts. Bmi1-positive ISCs are always maintained in a quiescent status if the intestine is not attacked. Bmi1-positive ISCs within crypts are activated soon after receiving $12 \mathrm{~Gy}$ abdominal irradiation as compensation for the tremendous loss of Lgr5-positive ISCs in a short time. ${ }^{32}$ However, ISCs gradually lose Bmi1 expression within 7 days after irradiation. ${ }^{32}$ In this study, we observed that Bmi1 expression was still present on cryptal cells 10 days after hAdMSC delivery, suggesting that the presence of Bmi1-positive ISCs was prolonged by hAd-MSCs. Moreover, hAd-MSC treatment significantly increased the number of Bmi1-positive cells in crypts on the 10th day after abdominal irradiation. Bmi1positive ISCs can convert into Lgr5-positive ISCs in vitro and in vivo. ${ }^{14,32}$ We observed an increase in proliferative cells within crypts after hAd-MSC delivery. These proliferative cells might have originated from the Lgr5-positive ISCs, ${ }^{31}$ because the number of Lgr5-positive ISCs in irradiated intestine rapidly increases after transplantation of bone marrow stromal cells. ${ }^{19}$ In this study, we believe that the Bmi1-positive ISCs were predominant in accelerating epithelial proliferation under threatening circumstances. Elevated levels of Bmi1-positive ISCs and their prolonged existence within crypts both contributed to the increasing numbers of Lgr5-positive ISCs, which subsequently developed into new villus-crypt structures to accelerate the restoration of epithelial integrity, and thus improved the function of the mucosal barrier in wounded intestine. ${ }^{33}$ Together with the hypertrophic villus, the absorptive areas of the intestines were enlarged, which temporarily compensated for the loss of function in the injured sites.

Several benefits of hAd-MSCs on RIII are described above. In the present study, homing of hAd-MSCs to injured sites was observed, and the mechanisms involved were preliminarily explored. However, the precise roles, direct or indirect, played by hAd-MSCs in wound healing deserve further discussion. Although adipose-derived MSCs can differentiate into epithelial cells and ECs, ${ }^{34,35}$ we believe that direct differentiation of hAd-MSCs into injured cells was not possible in the present study due to the species barrier. A previous study ${ }^{36}$ reported that rapid clearance of donor MSCs occurred soon after cell transplantation, but enhanced tissue repair was still observed. Thus, we conclude that the systemic responses triggered by hAd-MSCs were predominant in accelerating tissue repair. However, Wakabayashi et al. ${ }^{37}$ reported that human MSCs can induce the secretion of host VEGF and bFGF, mainly through paracrine signalling, to facilitate vascular repair in ischaemic mice. In this study, we also demonstrated that the expression of VEGF, bFGF and EGF within irradiated intestine were upregulated on the 10th day after abdominal irradiation in the context of hAd-MSCs delivery. High levels of these growth factors within injured sites facilitated tissue repair, especially in the proliferation of ISCs and ECs. On the other hand, human MSCs promote the mobilisation of murine endothelial lineage cells to injured sites to promote the regeneration of blood vessels. ${ }^{29}$ In the present study, a large number of CD31-positive HSCs/HPCs were found within the injured sites after the delivery of hAd-MSCs. Homing of HSCs to the ischaemic area is mediated by the interaction between SDF-1 and CXCR4. ${ }^{38}$ We evaluated SDF-1 expression within the injured sites and found that delivery of hAdMSCs further increased the local level of SDF-1 compared with the groups without hAd-MSC delivery. This was most probably due to the high level of SDF-1, which triggered and accelerated the mobilisation of CD31-positive HSCs/HPCs to ischaemic areas. These mechanisms deserve further research.

In summary, the delivery of a minimum number of hAdMSCs resulted in great therapeutic effects in the present study. As previously described, ${ }^{22}$ MSCs homed to the injured sites to signal to local cells involved in mitigating inflammation and preserving organ function.

\section{Conclusion}

In the present study, several benefits of hAd-MSCs in healing RIII were revealed, including anti-inflammation, accelerating neovascularisation and promoting the restoration of epithelial integrity. To a great extent, these benefits were attributed to the regenerative response of the host, which was activated by hAd-MSCs. Overall, our results indicate that hAd-MSCs have therapeutic potential for RIII management.

\section{Materials and Methods}

Animals. Adult Sprague-Dawley rats, weighing $400-450 \mathrm{~g}$, were provided by the Laboratory Animal Center of the Academy of Military Medical Sciences (China). All animals were used according to good animal practices, and all animal experiments were approved by our local animal care and use committee. 
Models of RIII. Rats were anaesthetised with an intraperitoneal injection of $10 \%$ chloral hydrate $(0.6 \mathrm{ml} / 100 \mathrm{~g})$ and placed on a platform to receive a lethal dose of 15 Gy. The irradiated field was $5 \times 5 \mathrm{~cm}^{2}$ from the xiphoid process to the pubic symphysis. The real-time dose rate was $1.75 \mathrm{~Gy} / \mathrm{min}$ ( $160 \mathrm{kV}, 25 \mathrm{~mA}$, using a RS-2000 Pro Biological Irradiator, Rad-Source, Suwanee, GA, USA). After irradiation, rats were weighed every 2 days.

Cell culture. Fat tissue was kindly provided by a healthy, male human donor with their approval. As described in Cao et al., ${ }^{35}$ the cells were separated by a 200-mesh strainer and suspended in complete medium containing 90\% DMEMLG/F-12 (Gibco, Grand island, NY, USA) and 10\% FBS (Gibco). Thereafter, the cells were plated into a flask at a density of $2 \times 10^{4} / \mathrm{cm}^{2}$ and grown at $37^{\circ} \mathrm{C}$ with $5 \% \mathrm{CO}_{2}$ in a humidified atmosphere. Passaging was performed every 2-3 days. The cells were harvested at passage 6 for phenotypic identification. After abdominal irradiation, $5 \times 10^{6}$ cells were injected intraperitoneally within $2 \mathrm{~h}$. To detect the specific effects of hAd-MSCs on healing RIII, the same number of passage 6 human skin fibroblasts were also intraperitoneally injected within $2 \mathrm{~h}$ after abdominal irradiation. Primary fibroblasts were purchased from the cell bank of the Chinese Academy of Sciences and cultured in DMEM (Gibco) containing $10 \%$ FBS (Gibco) and grown at $37^{\circ} \mathrm{C}$ with $5 \% \mathrm{CO}_{2}$ in a humidified atmosphere. Passaging was performed every 5-6 days, and cells at passage 6 were harvested for delivery (Supplementary Figure 2).

Flow cytometry. Mouse anti-human CD11b-PE, CD19-FITC, CD34-FITC, CD45-PE, CD73-PE, CD90-PE, CD105-PE and HLA-DR-PE were used to identify the MSC phenotypes. Mouse IgG1-FITC and PE were used for isotype control. All antibodies were purchased from BD Biosciences (Franklin Lakes, NJ, USA).

Differentiation of hAd-MSCs. Cells were harvested at passage 6 and plated into six-well plates. Complete medium was added into each well for cell adherence to plastic. Twenty-four hours later, the medium from three wells was replaced with adipogenic (Gibco), osteogenic (Gibco) or chondrogenic (Gibco) medium, respectively. Cells in the remaining wells served as controls. Every 2-3 days, the medium was replaced according to the manufacturer's instructions. After 21 days, the cells were harvested for analysis. To identify adipocytes, intracellular fat droplets were stained by Oil Red $\mathrm{O}$. To identify osteoblasts, calcium in the cells was stained by Alizarin Red. Collagens were stained by toluidine blue to identify chondrocytes.

Tracing experiment. The cell tracker CM-Dil (Invitrogen Inc., Carlsbad, CA, USA) was dissolved in DMSO (Invitrogen) and applied to passage 6 cells for $30 \mathrm{~min}$ at $37^{\circ} \mathrm{C}$, as recommended by the manufacturer. After incubation, red fluorescence was detected using a fluorescence microscope (Olympus, Tokyo, Japan). Adherent cells were then digested, and the suspension was centrifuged (1000 r.p.m.) for $5 \mathrm{~min}$ and washed in PBS (pH 7.4). A total of $5 \times 10^{6}$ cells were intraperitoneally injected into irradiated and normal rats.

Serum IL-10 quantification. After abdominal irradiation, $0.5 \mathrm{ml}$ of peripheral blood was obtained from the caudal vein and serum was obtained by centrifugation. The rat IL-10 ELISA Kit (eBioscience, San Diego, CA, USA) was used to measure serum IL-10.

Pathological staining. All samples were harvested from irradiated intestines. Samples were fixed in 10\% neutral-buffered formalin for more than $12 \mathrm{~h}$, and then dehydrated and embedded with paraffin. Sections of $4 \mu \mathrm{m}$ were used for H\&E and other staining.

Immunohistochemistry and immunofluorescence. Paraffin-embedded sections were used for $\mathrm{IHC}$ and immunofluorescence (IF) analyses. First, sections were dewaxed and rehydrated. Next, the sections were immersed in Tris/EDTA (pH 9.0) and incubated for $15 \mathrm{~min}$ at $98^{\circ} \mathrm{C}$ for antigen retrieval. The sections were incubated with serum from the host for $30 \mathrm{~min}$ at room temperature to block nonspecific antigen-binding sites. Then, the primary antibody, which was diluted as recommended by the manufacturer, was added to the sections and incubated overnight at $4^{\circ} \mathrm{C}$. Unbound antibodies were washed away with PBS. For $\mathrm{IHC}$ analysis, sections were incubated in $0.3 \% \mathrm{H}_{2} \mathrm{O}_{2}$ for $15 \mathrm{~min}$ to block endogenous peroxidase. Secondary antibody was then added and incubated at $37^{\circ} \mathrm{C}$ for $2 \mathrm{~h}$. If the sections were used for IHC analysis, DAB was added to detect positive cells. For IF analysis, the sections were directly imaged under microscope.
Primary antibodies used for IF included CD31 (Abcam, Cambridge, MA, USA), vWF (Abcam), CD34 (Santa Cruz Biotechnology, Santa Cruz, CA, USA), CD105 (Santa Cruz Biotechnology), Flk-1 (Santa Cruz Biotechnology) and CD133 (Abnova Biotechnology, Taiwan). Bmi1 antibody (Abcam) was used for IHC analysis. Ki-67 antibody (Abcam) was used for both IHC and IF. Secondary antibodies used for IF were Alexa Fluor 488 (Invitrogen), Alexa Fluor 594 (Invitrogen) and Alexa Fluor 633 (Invitrogen). An IHC staining kit (Abcam) was used to detect Ki-67 and Bmi1.

As described in Campos et al., ${ }^{39}$ FITC-labelled Griffonia simplicifolia isolectin-B4 (Invitrogen) was used to examine the vasculature. Briefly, a 1:100 dilution of a $1 \mu \mathrm{g} / \mu \mathrm{l}$ solution of isolectin-B4 was prepared and incubated with the sections overnight at $4^{\circ} \mathrm{C}$.

Real-time PCR. For real-time PCR, $100 \mathrm{mg}$ of irradiated intestine was freshly isolated and immersed in $1 \mathrm{ml}$ of RNA-later reagent (Invitrogen) for $30 \mathrm{~min}$. Next, total RNA was isolated using TRIzol (Invitrogen), and $1 \mu \mathrm{g}$ of total RNA from each sample was used for synthesis of first-strand CDNA using the RT-PCR Kit (Takarabio Inc., Shiga, Japan). Total CDNA was then added with primers for rat EGF, VEGF, bFGF, Flk-1, SDF-1, VCAM-1 and ICAM-1. In addition, beta-actin primers were used as controls. The sequences of these primers were as follows:

VEGF: $5^{\prime}$-GCTGCAATGATGAAGCCCTG-3' (Forward) and 5'-AGGCTCAC AGTGAATGTGGT-3' (Reverse); bFGF: $5^{\prime}$-AAAACCTGACCCGATCCCTC-3' (Forward) and 5'-AGAATCTGTCCCGTTCGGC-3' (Reverse); Flk-1: 5'-TACCA TAGCGTGACGAGCAG-3' (Forward) and5'-GCTGCTCAGACCTCACAGTC-3' (Reverse); EGF: $5^{\prime}$-CGTCTTTAGCCCCATCCCTC-3' (Forward) and $5^{\prime}$-TCTG ACCCCCAGAACTTCCA-3' (Reverse); SDF-1: 5'-ACGGTCTTGAACTACTG GCG-3' (Forward) and 5'-GGAGGCTTACAGCACGAAAC-3' (Reverse); VCAM-1: $5^{\prime}$-TGGGGAGGTGAGGGATGAAG-3' (Forward) and 5'-ACACATTAGG GACCGTGCAG-3' (Reverse); ICAM-1: 5'-ACCCACCTCACAGGGTACTT-3' (Forward) and $5^{\prime}$-TCTTGTCCAGGTGAGGACCA-3' (Reverse); beta-actin: $5^{\prime}$-ACC CGCGAGTACAACCTTC-3' (Forward) and 5'-CCATACCCACCATCACACCC-3' (Reverse). Quantitative PCRs were performed using the SYBR Green I TaqMan probes (Roche, Basel, Switzerland) in 40 amplification cycles in an ABI 7500 Fast.

Statistical analysis. The data were analysed using SPSS 17.0 software (SPSS Inc., Chicago, IL, USA) and expressed as mean \pm S.D. Animal survival curves were analysed using the Kaplan-Meier method. One-way ANOVA was performed to compare data among groups. Statistical significance was defined as $P \leq 0.05$

\section{Conflict of Interest}

The authors declare no conflict of interest.

1. Potten CS, Booth $C$. The role of radiation-induced and spontaneous apoptosis in the homeostasis of the gastrointestinal epithelium: a brief review. Comp Biochem Physiol B Biochem Mol Biol 1997; 118: 473-478.

2. Umar S. Intestinal stem cells. Curr Gastroenterol Rep 2010; 12: 340-348.

3. Wang J, Boerma M, Fu Q, Hauer-Jensen M. Significance of endothelial dysfunction in the pathogenesis of early and delayed radiation enteropathy. World J Gastroenterol 2007; 13: 3047-3055.

4. Kountouras J, Zavos C. Recent advances in the management of radiation colitis. World $J$ Gastroenterol 2008; 14: 7289-7301.

5. Semont A, Mouiseddine M, Francois A, Demarguay C, Mathieu N, Chapel A et al. Mesenchymal stem cells improve small intestinal integrity through regulation of endogenous epithelial cell homeostasis. Cell Death Differ 2010; 17: 952-961.

6. Jiang $Y$, Jahagirdar BN, Reinhardt RL, Schwartz RE, Keene CD, Ortiz-Gonzalez XR et al. Pluripotency of mesenchymal stem cells derived from adult marrow. Nature 2002; 418: 41-49.

7. Dominici M, Le Blanc K, Mueller I, Slaper-Cortenbach I, Marini F, Krause D et al. Minimal criteria for defining multipotent mesenchymal stromal cells. The International Society for Cellular Therapy position statement. Cytotherapy 2006; 8: 315-317.

8. Lee RH, Kim B, Choi I, Kim H, Choi HS, Suh K et al. Characterization and expression analysis of mesenchymal stem cells from human bone marrow and adipose tissue. Cell Physiol Biochem 2004; 14: 311-324.

9. Sanchez L, Gutierrez-Aranda I, Ligero G, Rubio R, Munoz-Lopez M, Garcia-Perez JL et al. Enrichment of human ESC-derived multipotent mesenchymal stem cells with immunosuppressive and anti-inflammatory properties capable to protect against experimental inflammatory bowel disease. Stem Cells 2011; 29: 251-262.

10. Ji JF, He BP, Dheen ST, Tay SS. Interactions of chemokines and chemokine receptors mediate the migration of mesenchymal stem cells to the impaired site in the brain after hypoglossal nerve injury. Stem Cells 2004; 22: 415-427. 
11. Ren G, Zhao X, Zhang L, Zhang J, L'Huillier A, Ling W et al. Inflammatory cytokine-induced intercellular adhesion molecule-1 and vascular cell adhesion molecule-1 in mesenchymal stem cells are critical for immunosuppression. J Immunol 2010; 184: 2321-2328.

12. Abderrahmani R, Francois A, Buard V, Tarlet G, Blirando K, Hneino M et al. PAI-1. dependent endothelial cell death determines severity of radiation-induced intestinal injury. PLoS One 2012; 7: e35740.

13. Kim H, Cho HJ, Kim SW, Liu B, Choi YJ, Lee J et al. CD31 + cells represent highly angiogenic and vasculogenic cells in bone marrow: novel role of nonendothelial CD31+ cells in neovascularization and their therapeutic effects on ischemic vascular disease. Circ Res 2010; 107: 602-614.

14. Tian H, Biehs B, Warming S, Leong KG, Rangell L, Klein OD et al. A reserve stem cell population in small intestine renders Lgr5-positive cells dispensable. Nature 2011; 478 255-259.

15. Roth S, Franken P, Sacchetti A, Kremer A, Anderson K, Sansom O et al. Paneth cells in intestinal homeostasis and tissue injury. PLoS One 2012; 7: e38965.

16. Sato T, van Es JH, Snippert HJ, Stange DE, Vries RG, van den Born M et al. Paneth cells constitute the niche for Lgr5 stem cells in intestinal crypts. Nature 2011; 469: 415-418.

17. Paris F, Fuks Z, Kang A, Capodieci P, Juan G, Ehleiter D et al. Endothelial apoptosis as the primary lesion initiating intestinal radiation damage in mice. Science 2001; 293: 293-297.

18. Johnson LB, Riaz AA, Adawi D, Wittgren L, Back S, Thornberg C et al. Radiation enteropathy and leucocyte-endothelial cell reactions in a refined small bowel model. BMC Surg 2004; 4: 10.

19. Saha S, Bhanja P, Kabarriti R, Liu L, Alfieri AA, Guha C. Bone marrow stromal cell transplantation mitigates radiation-induced gastrointestinal syndrome in mice. PLoS One 2011; 6: e24072.

20. Kudo K, Liu Y, Takahashi K, Tarusawa K, Osanai M, Hu DL et al. Transplantation of mesenchymal stem cells to prevent radiation-induced intestinal injury in mice. J Radiat Res 2010; 51: 73-79.

21. Hakansson A, Molin G. Gut microbiota and inflammation. Nutrients 2011; 3: 637 .

22. Auletta JJ, Deans RJ, Bartholomew AM. Emerging roles for multipotent, bone marrowderived stromal cells in host defense. Blood 2012; 119: 1801-1809.

23. Langberg CW, Sauer T, Reitan JB, Hauer-Jensen M. Relationship between intestina fibrosis and histopathologic and morphometric changes in consequential and late radiation enteropathy. Acta Oncol 1996; 35: 81-87.

24. Yagi H, Soto-Gutierrez A, Parekkadan B, Kitagawa Y, Tompkins RG, Kobayashi $\mathrm{N}$ et al. Mesenchymal stem cells: mechanisms of immunomodulation and homing. Cell Transplant 2010; 19: 667-679.

25. Aggarwal S, Pittenger MF. Human mesenchymal stem cells modulate allogeneic immune cell responses. Blood 2005; 105: 1815-1822.

26. Nemeth K, Leelahavanichkul A, Yuen PS, Mayer B, Parmelee A, Doi K et al. Bone marrow stromal cells attenuate sepsis via prostaglandin $\mathrm{E}(2)$-dependent reprogramming of host macrophages to increase their interleukin-10 production. Nat Med 2009; 15: 42-49.

27. Gonzalez-Rey E, Anderson P, Gonzalez MA, Rico L, Buscher D, Delgado M. Human adult stem cells derived from adipose tissue protect against experimental colitis and sepsis. Gut 2009; 58: 929-939.
28. Brittan M, Chance V, Elia G, Poulsom R, Alison MR, MacDonald TT et al. A regenerative role for bone marrow following experimental colitis: contribution to neovasculogenesis and myofibroblasts. Gastroenterology 2005; 128: 1984-1995.

29. Chen L, Tredget EE, Wu PY, Wu Y. Paracrine factors of mesenchymal stem cells recruit macrophages and endothelial lineage cells and enhance wound healing. PLoS One 2008; 3: e1886.

30. Stout RD, Suttles J. Immunosenescence and macrophage functional plasticity: dysregulation of macrophage function by age-associated microenvironmental changes. Immunol Rev 2005; 205: 60-71.

31. Barker N, van Es JH, Kuipers J, Kujala P, van den Born M, Cozijnsen M et al. Identification of stem cells in small intestine and colon by marker gene Lgr5. Nature 2007; 449 : 1003-1007.

32. Yan KS, Chia LA, Li X, Ootani A, Su J, Lee JY et al. The intestinal stem cell markers Bmi1 and Lgr5 identify two functionally distinct populations. Proc Natl Acad Sci USA 2012; 109: 466-471.

33. Sato T, Vries RG, Snippert HJ, van de Wetering M, Barker N, Stange DE et al. Single Lgr5 stem cells build crypt-villus structures in vitro without a mesenchymal niche. Nature 2009; 459: 262-265.

34. Ando Y, Inaba M, Sakaguchi Y, Tsuda M, Quan GK, Omae M et al. Subcutaneous adipose tissue-derived stem cells facilitate colonic mucosal recovery from 2,4,6-trinitrobenzene sulfonic acid (TNBS)-induced colitis in rats. Inflamm Bowel Dis 2008; 14: 826-838.

35. Cao Y, Sun Z, Liao L, Meng Y, Han Q, Zhao RC. Human adipose tissue-derived stem cells differentiate into endothelial cells in vitro and improve postnatal neovascularization in vivo. Biochem Biophys Res Commun 2005; 332: 370-379.

36. Lange C, Brunswig-Spickenheier B, Cappallo-Obermann H, Eggert K, Gehling UM, Rudolph $\mathrm{C}$ et al. Radiation rescue: mesenchymal stromal cells protect from lethal irradiation. PLoS One 2011; 6: e14486.

37. Wakabayashi K, Nagai A, Sheikh AM, Shiota Y, Narantuya D, Watanabe T et al. Transplantation of human mesenchymal stem cells promotes functional improvement and increased expression of neurotrophic factors in a rat focal cerebral ischemia model. J Neurosci Res 2010; 88: 1017-1025.

38. Askari AT, Unzek S, Popovic ZB, Goldman CK, Forudi F, Kiedrowski M et al. Effect of stromal-cell-derived factor 1 on stem-cell homing and tissue regeneration in ischaemic cardiomyopathy. Lancet 2003; 362: 697-703.

39. Campos M, Amaral J, Becerra SP, Fariss RN. A novel imaging technique for experimental choroidal neovascularization. Invest Ophthalmol Vis Sci 2006; 47: 5163-5170.

(i) () $\Theta$ Cell Death and Disease is an open-access journal cy licensed under a Creative Commons Attribution-NonCommercialNoDerivs 3.0 Unported License. To view a copy of this license, visit http://creativecommons.org/licenses/by-nc-nd/3.0/

Supplementary Information accompanies this paper on Cell Death and Disease website (http://www.nature.com/cddis) 